\title{
Determining Rouse relaxation times from the dynamic modulus of entangled polymers
}

\author{
C. M. Roland ${ }^{\mathrm{a})}$ \\ Naval Research Laboratory, Chemistry Division, Code 6120, \\ Washington, DC 20375-5342 \\ L. A. Archer \\ Cornell University, School of Chemical Engineering, Ithaca, \\ New York 14853-5201 \\ P. H. Mott \\ Naval Research Laboratory, Chemistry Division, Code 6120, \\ Washington, DC 20375-5342 \\ J. Sanchez-Reyes \\ Cornell University, School of Chemical Engineering, Ithaca, \\ New York 14853-5201
}

(Received 30 July 2003; final revision received 14 November 2003)

\section{Synopsis}

Different methods of finding the Rouse relaxation times $\tau_{e}$, demarcating the onset of entanglement effects, were evaluated for entangled solutions of high molecular weight polystyrene in diethyl phthalate. Five expressions were utilized, involving variously the zero shear viscosity, the storage modulus at frequencies just beyond the rubbery plateau, the terminal Andrade creep region in the retardation spectrum, and the recoverable creep compliance. Values of $\tau_{e}$ determined by a procedure which assumes that the deformation modes are additive in the strain were substantially larger than the Rouse times extracted from the storage modulus by assuming stress additivity. (C) 2004 The Society of Rheology. [DOI: 10.1122/1.1645516]

This note evaluates different methods for determining the Rouse relaxation time $\tau_{e}$ beyond which entanglements constrain the dynamics, for an entangled polymer solution. It is motivated by the importance of Rouse contributions to the nonlinear relaxation dynamics of entangled polymer liquids, as well as the general significance of the Rouse model to theories of polymer relaxation. Our main objective is to compare predictions of $\tau_{e}$ obtained using deconvolution schemes based on additivity of the compliance (or strain) to results based on additivity of the modulus (or stress). For a relaxation spectrum comprised of weakly overlapping modes, the two approaches are expected to yield comparable results. However, as is often the case, the modes overlap whereby the method of deconvolution can influence the results. There is literature strongly advocating additivity

$\overline{{ }^{a)} \text { Electronic mail: roland@nrl.navy.mil }}$ 
TABLE I. Comparison of different determination of the longest Rouse relaxation $\tau_{\mathrm{R}}$ and retardation $\lambda_{\mathrm{R}}$ times for entangled solutions of polystyrene in diethylphthalate.

\begin{tabular}{|c|c|c|c|c|c|c|c|c|}
\hline \multirow[b]{2}{*}{$\begin{array}{l}\text { Solution } \\
(\%)\end{array}$} & \multirow[b]{2}{*}{$\begin{array}{c}M_{w} \times 10^{-6} \\
\text { (Daltons) }\end{array}$} & \multirow[b]{2}{*}{$M_{w} / M_{n}$} & \multirow[b]{2}{*}{$\phi$} & \multicolumn{3}{|c|}{$\tau_{e}(\mathrm{~s})$} & \multicolumn{2}{|c|}{$\lambda_{e}(\mathrm{~s})$} \\
\hline & & & & $\begin{array}{l}\text { Eqs. (1) } \\
\text { and (2) }\end{array}$ & $\begin{array}{l}\text { Eqs. (2) } \\
\text { and (3) }\end{array}$ & Eq. (4) & Eq. (5) & Eq. (6) \\
\hline PS3.8M6 & 3.84 & 1.04 & 0.06 & $3 \times 10^{-3}$ & $1 \times 10^{-3}$ & $9 \times 10^{-4}$ & $0.016 \pm 0.01$ & - \\
\hline PS5.48M6 & 5.48 & 1.15 & 0.058 & $4 \times 10^{-3}$ & $9 \times 10^{-4}$ & $9 \times 10^{-4}$ & $0.027 \pm 0.01$ & - \\
\hline PS8.4M6 & 8.42 & 1.2 & 0.067 & $3 \times 10^{-3}$ & $7 \times 10^{-4 a}$ & $7 \times 10^{-4 b}$ & $0.026 \pm 0.03$ & $0.02 \pm 0.002$ \\
\hline PS20M8 & 20.07 & 1.2 & 0.079 & $9 \times 10^{-4}$ & $1 \times 10^{-3}$ & $1 \times 10^{-3}$ & $0.50 \pm 0.05$ & $0.56 \pm 0.02$ \\
\hline
\end{tabular}

${ }^{\mathrm{a}}$ The value of $\tau_{\mathrm{R}}$ was incorrectly reported as $0.5 \mathrm{~s}$ in the publication of Sanchez-Reyes and Archer (2003).

${ }^{\mathrm{b}}$ The original estimate was $\tau_{e} \sim 3.6 \times 10^{-4} \mathrm{~s}$ [Sanchez-Reyes and Archer (2003)].

of the compliance functions [Dean et al. (1990); Plazek (1992); Mott and Roland (1998); Roland and Mott (1999); Plazek and Echeverria (2000)], although the alternate approach has been adopted by various workers [Read (1983); Okamoto et al. (1995); SanchezReyes and Archer (2003)]. We find herein for entangled solutions of high-molar mass polystyrenes (PS) in diethyl phthalate (Table I) that the entanglement Rouse relaxation time obtained assuming stress additivity is at least an order of magnitude smaller that $\tau_{e}$ obtained using strain additivity.

The Doi and Edwards (1986) constitutive theory for entangled polymer rheology contends that a polymeric liquid relaxes stress in two stages. At short times following deformation, entangled molecules equilibrate their contour length by Rouse dynamic processes, while at long times reptation randomizes any orientation of segments produced by the deformation. This clear separation between the time scales on which molecules relax orientation and length carries over to all constitutive theories for entangled linear polymers based on the tube model. In these theories, the average molecular orientation relaxation time is the reptation time, while the contour length relaxation time is taken to be the longest Rouse relaxation time $\tau_{\mathrm{R}}$. In principle, this assumption can be evaluated from stress growth or stress relaxation experiments in steady shear or following step shear deformations [Einaga et al. (1971); Osaki et al. (1982); Archer (1999); SanchezReyes and Archer (2002), (2003)]. Unfortunately, the longest Rouse relaxation time of entangled polymers is not easily obtained from such experiments, impeding rigorous comparisons. Indeed, the Rouse model per se breaks down upon the onset of entanglement effects at $\tau_{e}$.

In the absence of data for the viscoelastic spectrum other than the zero-shear viscosity $\eta_{0}$, an estimate of $\tau_{\mathrm{R}}$ can be found from the relationship between $\eta_{0}$ and polymer molecular weight [Menezes and Graessley (1982)]:

$$
\tau_{\mathrm{R}}=\frac{6 M_{\mathrm{w}}^{2-\mu}}{\pi^{2} \rho \mathrm{RT}} \frac{M_{\mathrm{c}}^{\mu-1}}{\phi^{4 / 3(\mu-1)+1}} \eta_{0} .
$$

Here $\mu$ is the scaling exponent of $\eta_{0}(=3.4 \pm 0.3)$ [Ferry (1980)], $M_{w}$ is the weight average molecular weight, $M_{c}$ the critical molecular weight at which entanglement effects become apparent $(\approx 31000$ Daltons for PS [Fetters et al. (1999)]), $\rho$ the solvent density, $\phi$ the polymer volume fraction in solution, and RT have their usual significance. This method can be in error by as much as a factor of ten [Osaki et al. (2000), (2001)], which is unsurprising, given the uncertainties in $M_{c}$ and the exponents. Using the usual Rouse scaling relation, $\tau_{\mathrm{R}} \sim M_{w}^{2}$, values of $\tau_{e}$ were calculated as 

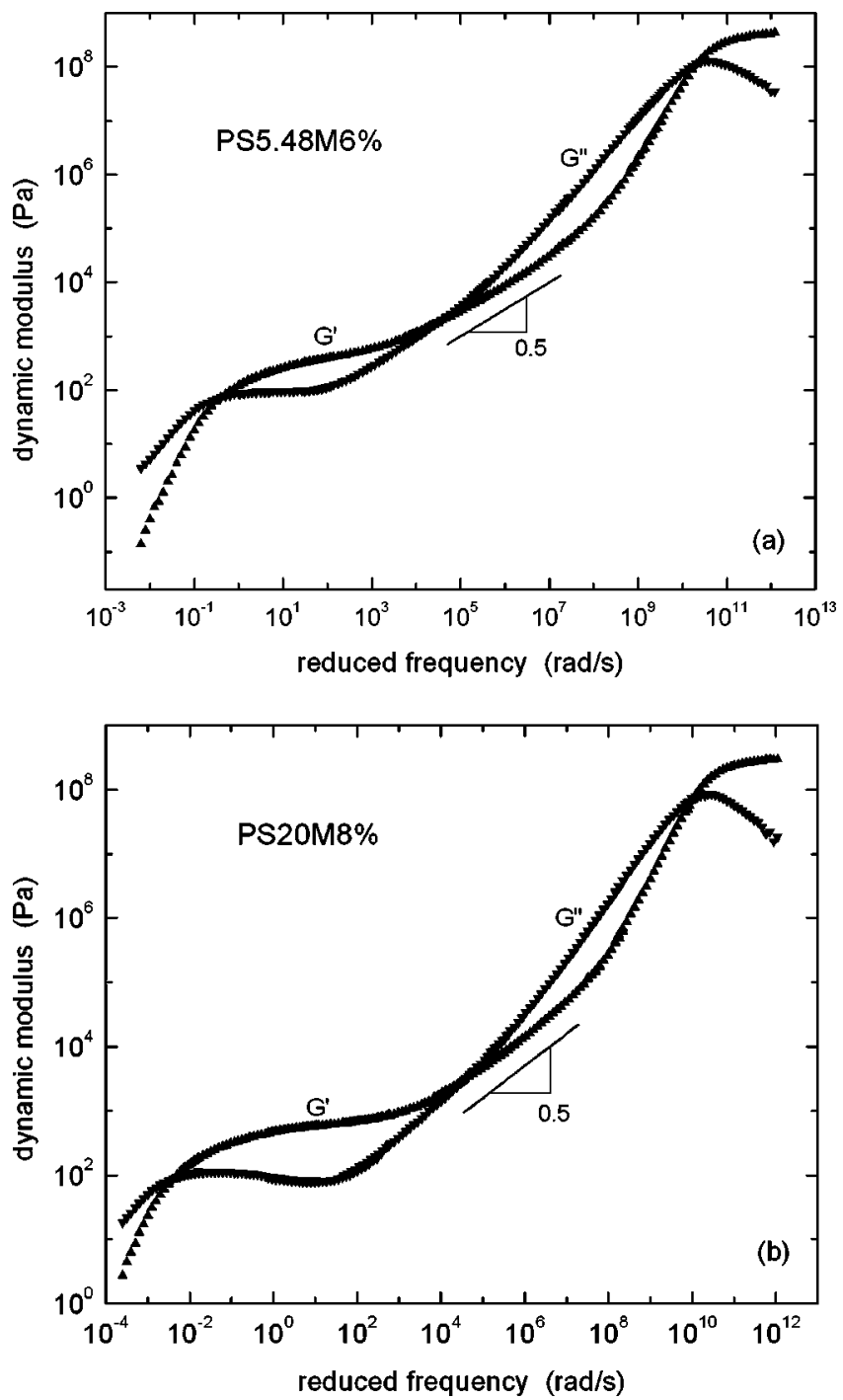

FIG. 1. Dynamic storage and loss modulus vs reduced frequency, for (a) $6 \%$ PS ( $5.48 \times 10^{6}$ Daltons) solution, and (b) $8 \%$ PS $\left(20.07 \times 10^{6}\right.$ Daltons) solution, in diethyl phthalate.

$$
\tau_{e}=\tau_{\mathrm{R}}\left(M_{e} / M_{w}\right)^{1 / 2}
$$

with the molecular weight between entanglements $M_{e}$ taken to vary as the $-4 / 3$ power of the polymer volume fraction. (Generally, this exponent is found to range from -1 to -1.5 [Tao et al. (1999)]). For neat PS, $M_{e}=18100 \mathrm{D}$ [Fetters et al. (1999)]. The results are listed in Table I for the four entangled solutions of high molecular weight polystyrene (PS) in diethyl phthalate (DEP) studied by Sanchez-Reyes and Archer (2003).

Osaki and coworkers (2001) recently presented a simple approach for determining $\tau_{R}$ from high-frequency oscillatory shear rheology experiments. The storage modulus $G^{\prime}(\omega)$, at frequencies $\omega$ just beyond the rubbery plateau regime, is fitted to the Rouse modulus expression $G^{\prime}(\omega)=\alpha \omega^{1 / 2}$ [see Fig. 1(a)], and the longest Rouse relaxation time is found from 


$$
\tau_{\mathrm{R}}=\left(\frac{\alpha M_{w}}{1.11 c \mathrm{RT}}\right)^{2}
$$

where $c$ is the mass of polymer per unit volume of solution. The entanglement Rouse time is in turn calculated using Eq. (2). One problem with Osaki's procedure is that the classical Rouse scaling relationship, $G^{\prime}(\omega)=\alpha \omega^{1 / 2}$, is only observed over a limited range of frequencies. The prefactor $\alpha$ must then be determined from a relatively small amount of experimental data, reducing the accuracy of $\tau_{\mathrm{R}}$ or $\tau_{e}$ estimates. This problem arises from overlap of the local segmental and solvent contributions to the relaxation modulus with the Rouse chain modes. A second potential limitation of the method of Osaki is that some portion of the spectrum will always have the correct "Rouse" slope $\left(\sim \omega^{1 / 2}\right)$, since the strong frequency-dependence of the modulus in the transition zone must segue into the flat plateau region. Since it is not possible to evaluate a priori the origin of the narrow $\left(\sim \omega^{1 / 2}\right)$ scaling regime, the possibility exists for large errors.

Sanchez-Reyes and Archer (2003) recently suggested a procedure for removing solvent and segmental contributions from the dynamic moduli of entangled polymer solutions. In their approach, the high-frequency storage and loss moduli are first fitted using a multimode Maxwell model. The resulting Maxwell model expressions are used to calculate the segmental and solvent contributions, $G_{S}^{\prime}(\omega)$ and $G_{S}^{\prime \prime}(\omega)$, for the entire range of frequencies. Local segmental and solvent contributions are then removed by subtracting $G_{S}^{\prime}(\omega)$ and $G_{S}^{\prime \prime}(\omega)$ from the experimental $G^{\prime}(\omega)$ and $G^{\prime \prime}(\omega)$ data. This method is illustrated in Figs. 1(a) and 1(b), showing master curves of the dynamic mechanical moduli measured using two PS/DEP solutions (PS5.48M6 and PS20M8\%, see Table I) at a reference temperature of $28.5^{\circ} \mathrm{C}$. Figures 2(a) and 2(b) are the respective moduli obtained after subtracting the solvent and segmental contributions. It is clear from both the latter plots that the procedure yields dynamic moduli exhibiting Rouse scaling over a relatively broad frequency range. For the PS20M8\% sample, the storage modulus shows more deviation from the $\omega^{1 / 2}$ behavior. This may reflect some ambiguity in the shape of the curves due to the well-established breakdown of time-temperature superpositioning in the transition zone [Roland et al. (2001); Santangelo and Roland (1998); Plazek (1996); Plazek et al. (1995)]. The ratio $G^{\prime \prime} / G^{\prime}$ increases with decreasing temperature in this region of the spectrum, reflecting the overlap of the local segmental and the chain modes.

The longest Rouse relaxation time is determined by fitting the storage moduli in Figs. 2(a) and 2(b) to the expression $G^{\prime}(\omega)=\alpha \omega^{1 / 2}$, and using Eq. (3) to compute $\tau_{\mathrm{R}}$, with values of $\tau_{e}$ then obtained from Eq. (2). Results for the four solutions are provided in Table I. These estimates are generally close to the values obtained by fitting the raw storage modulus data [Fig. 1(a)], in the manner of Osaki and coworkers (2001).

Finally, there is a direct method to deduce $\tau_{e}$ from the modulus, as the intersection of the extrapolated rubber plateau $G_{N}$ with the extrapolation of the $\omega^{1 / 2}$ portion of the Rouse transition zone; thus,

$$
\tau_{e}=\left(\alpha / G_{n}\right)^{2}
$$

These estimates of $\tau_{e}$ are also given in the table.

The methods used by Osaki and coworkers (2001) and by Sanchez-Reyes and Archer (2003) for determining $\tau_{\mathrm{R}}$ assume that (i) a pure Rouse contribution can be isolated in at least a portion of the experimental $G^{\prime}(\omega)$ spectrums and (ii) the Rouse and local segmental contributions simply add in the modulus. If chain modes and the segmental relaxation modes are well-separated in frequency, the former assumption is reasonable. 

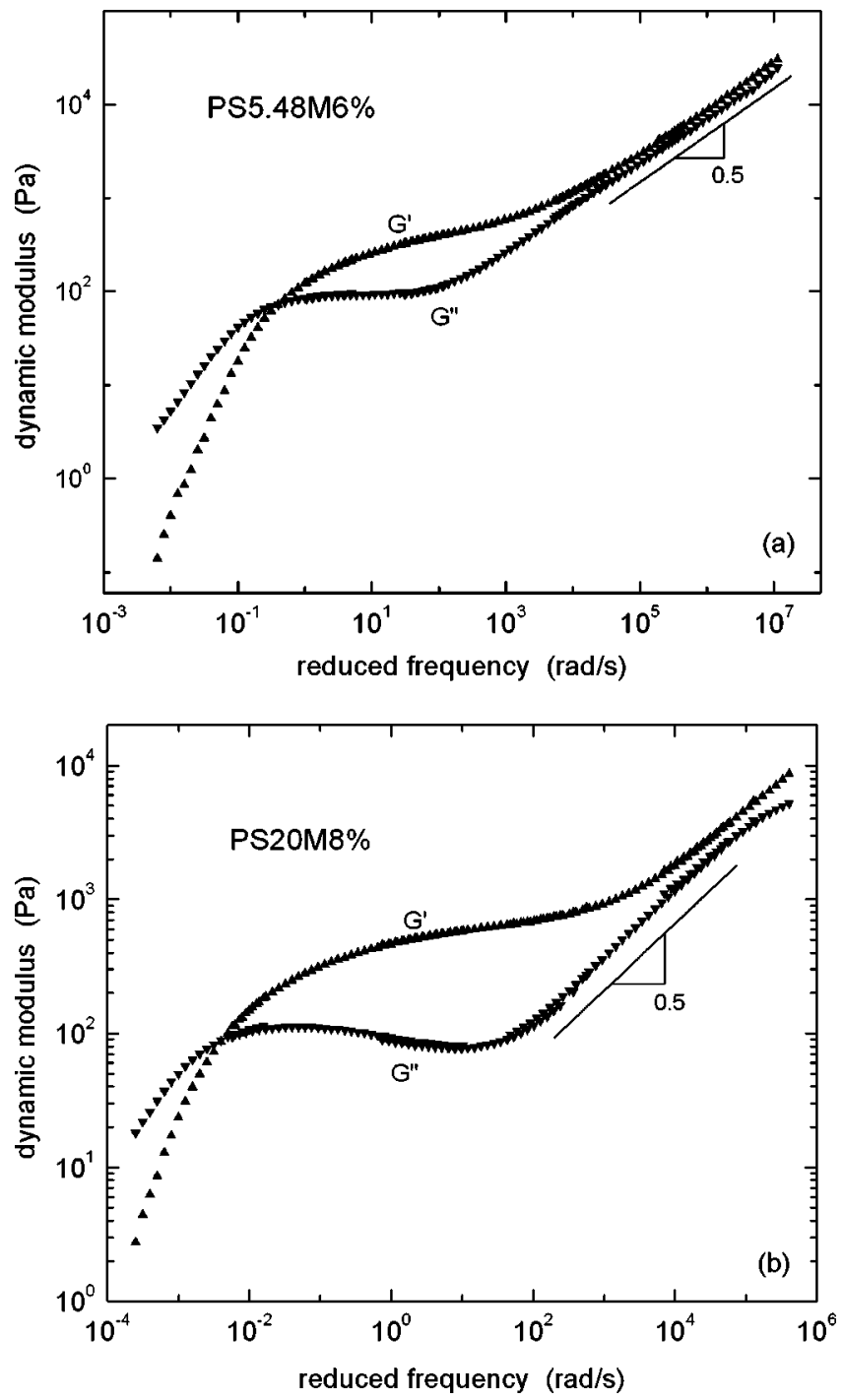

FIG. 2. Data in Fig. 1 after subtraction of solvent and local segmental contributions to the dynamic moduli: (a) $6 \%$ PS (5.48 $\times 10^{6}$ Daltons) solution, and (b) $8 \%$ PS (20.07 $\times 10^{6}$ Daltons) solution. The lines with the slope of $1 / 2$ represent the power law used to extract the Rouse behavior.

However, it is not obvious by inspection when sufficient separation has been attained. Moreover, the breakdown of time-temperature superpositioning in the transition zone belies such separation, since thermorheological complexity is a consequence of the differing temperature dependence of the two modes [Roland et al. (2001); Santangelo and Roland (1998); Plazek (1996); Plazek et al. (1995)]. Another intrusion of the chain modes into the high frequency dynamics comes from $\eta_{0}$, which can be shown to make a measurable contribution to the dynamic modulus even at the glass transition temperature [Plazek and O'Rourke (1971)]. Furthermore, from an analysis of stress birefringence data, Okamoto et al. (1995) concluded that the local segmental relaxation (referred to therein as the glassy component) dominates the viscoelastic response at frequencies well 
into the rubbery plateau region. Clearly, a satisfactory method of deconvoluting overlapping contributions to the viscoelastic spectrum is of quite general importance.

Many workers have analyzed their experimental data by assuming additivity of the strains, whereby the viscosity [Janeschitz-Kriegl (1983); Ferry (1980)], segmental relaxation [Mott and Roland (1998); Ngai et al. (1996)], glassy compliance [Plazek et al. (1994)] and solvent contributions [Plazek et al. (1979)] are removed by subtraction of the appropriate compliance functions. Collectively, these results argue that overlapping contributions to the viscoelastic spectrum are additive in the compliance, not the modulus. Certainly, in the case of the viscous deformation and recoverable strain, the compliance functions are additive, as directly verified by creep-recovery experiments [Ferry (1980); Laun (1981)].

The dynamic mechanical data for the four PS solutions were reanalyzed by calculating the retardation spectra $L(\lambda)$, obtained from the dynamic moduli using [Ferry (1980)]

$$
J^{\prime}(\omega)=J_{g}+\int_{-\infty}^{\infty} \frac{L}{1+\omega^{2} \lambda^{2}} d \ln \lambda,
$$

where $J_{g}$ is the instantaneous compliance of the glass, and the storage compliance $J^{\prime}(\omega)=G^{\prime} /\left(G^{\prime 2}+G^{\prime \prime 2}\right)$. Conversion of the spectra was carried out by a nonlinear regularization method [Honerkamp and Weese (1993)]; the results were internally selfconsistent. Figures 3(a) and 3(b) show the respective retardation spectra $L(\lambda)$ of PS8.4M6 and PS20M8\%. Both exhibit Rouse-mode and terminal maxima, which are separated by a region over which $L(\lambda) \propto \lambda^{1 / 3}$, the so-called Andrade terminal region [Andrade (1910); Plazek (1960); Berry (1976)]. (At shorter times, the solvent and segmental peaks are not well resolved. This may account for the absence of any "primary Andrade" creep region at shorter times, where again $L(\lambda) \propto \lambda^{1 / 3}$.) At longer times, the transition to the Andrade terminal behavior marks the onset of entangled dynamics, thereby defining of the Rouse retardation time, $\lambda_{e}$.

The obtained Rouse retardation times for all four solutions are listed in Table I. At fixed concentration, the entanglement time is expected to be independent of molecular weight [Ferry (1980)]. For the three solutions having the same concentration-PS3.8M6, PS5.48M6, and PS8.4M6\%- $\lambda_{e}$ are indeed constant to within the experimental error. This is also true for the $\tau_{e}$ in Table I. However, there is a substantial difference between the respective retardation and relaxation times, the former larger by an order of magnitude or more. The relation between the terminal retardation times and relaxation times of the Rouse model has been addressed by Berry (1987). At the long time end of the Rouse region (i.e., when the Rouse chain segment approaches the entanglement length), if the viscous contribution to the deformation is sufficiently small, the material can be treated as a Rouse solid, whereby $\tau_{1}=\tau_{e}$ and $\lambda_{0}=\lambda_{e}=2.38 \tau_{e}$ [Berry (1987)]. However, the $\lambda_{e}$ in Table I exceed $\tau_{e}$ by considerably more than this factor of 2.38 .

An alternative method of obtaining $\lambda_{e}$ is from the deviation of the recoverable creep compliance $J_{\mathrm{R}}(t)$ in the terminal region. The recoverable creep compliance is calculated from the retardation spectrum as

$$
J_{\mathrm{R}}(t)=J_{g}+\int_{-\infty}^{\infty} L\left(1-e^{-t / \lambda}\right) d \ln \lambda
$$

in which $t$ is the creep time. Since the terminal Andrade creep $J_{A}(t)$ follows the relation

$$
J_{\mathrm{A}}(t)=J_{\mathrm{A}}+\beta t^{1 / 3},
$$



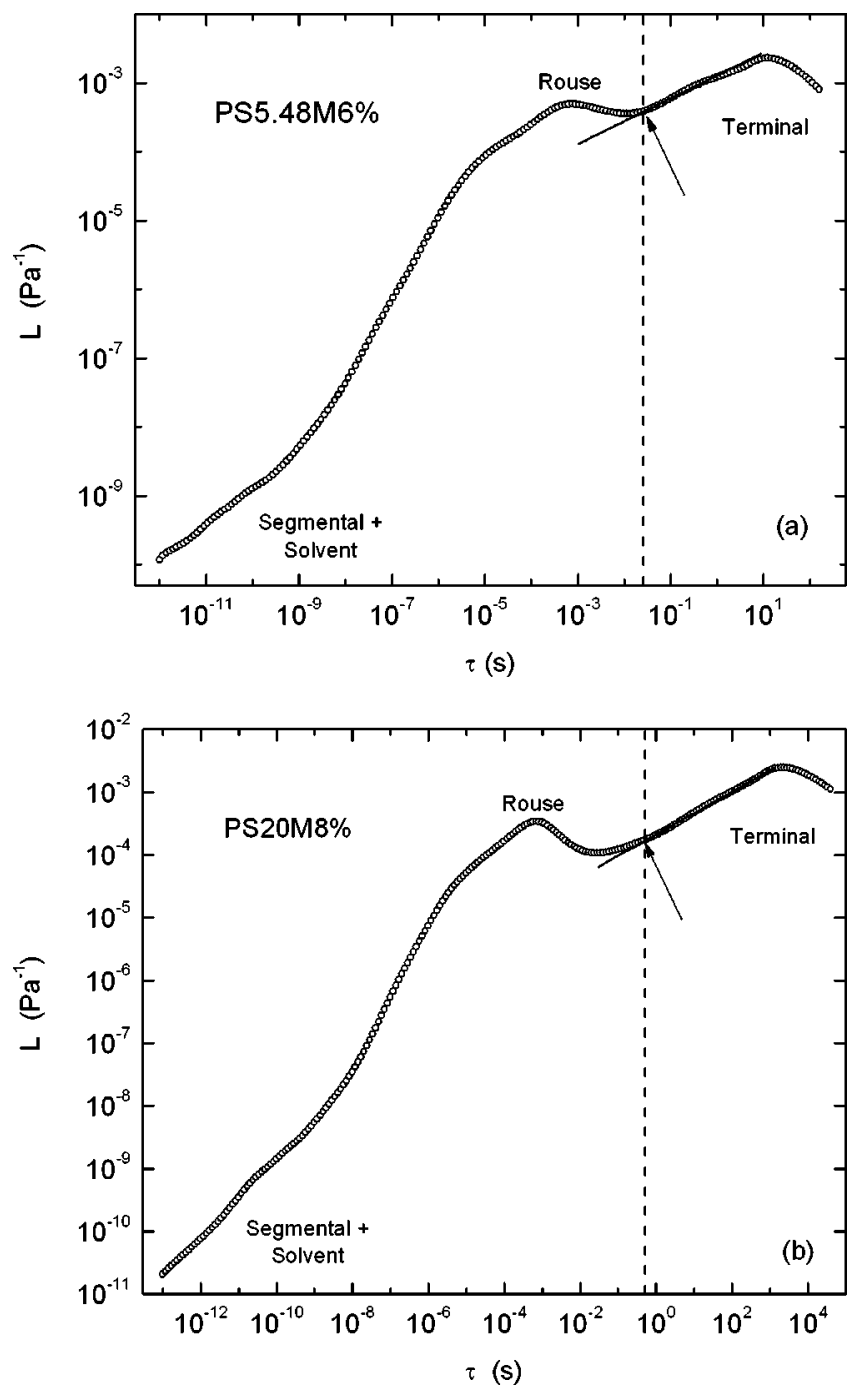

FIG. 3. Retardation spectrum for (a) $6 \%$ PS $\left(5.48 \times 10^{6}\right.$ Daltons) and (b) $8 \%$ PS $\left(20.07 \times 10^{6}\right.$ Daltons) in diethyl phthalate, calculated from the dynamic modulus in Fig. 1. The deviation from the Andrade fit, denoted by the dashed line at $\lambda=\lambda_{e}$, demarcates the onset of entanglement effects, separating the Rouse and terminal zones.

where $J_{\mathrm{A}}$ and $\beta$ are temperature-dependent constants, the onset of entanglements is revealed when $J_{\mathrm{R}}(t)$ becomes a straight line when plotted versus $t^{1 / 3}$ [Plazek (1960)]. The result is shown in Fig. 4, from which we obtain $\lambda_{e}=0.56 \pm 0.02 \mathrm{~s}$ for PS20M8\%. This is equal to within the experimental error to the determination from $L(\lambda)$, and again significantly larger than the $\tau_{e}$ from Eqs. (1)-(4). Similar results are obtained for PS8.4M6\% (Table I). The two solutions of the lower molecular weight PS yield Andrade regions of insufficient extent for a reliable assessment to be made.

In conclusion, different procedures were used to deduce entanglement Rouse relaxation (or retardation) times from dynamic mechanical measurements. Values of $\lambda_{e}$ obtained by rigorously deconvoluting the retardation spectrum are consistently larger than the $\tau_{e}$ obtained from analysis of the modulus functions, although both methods yield the 


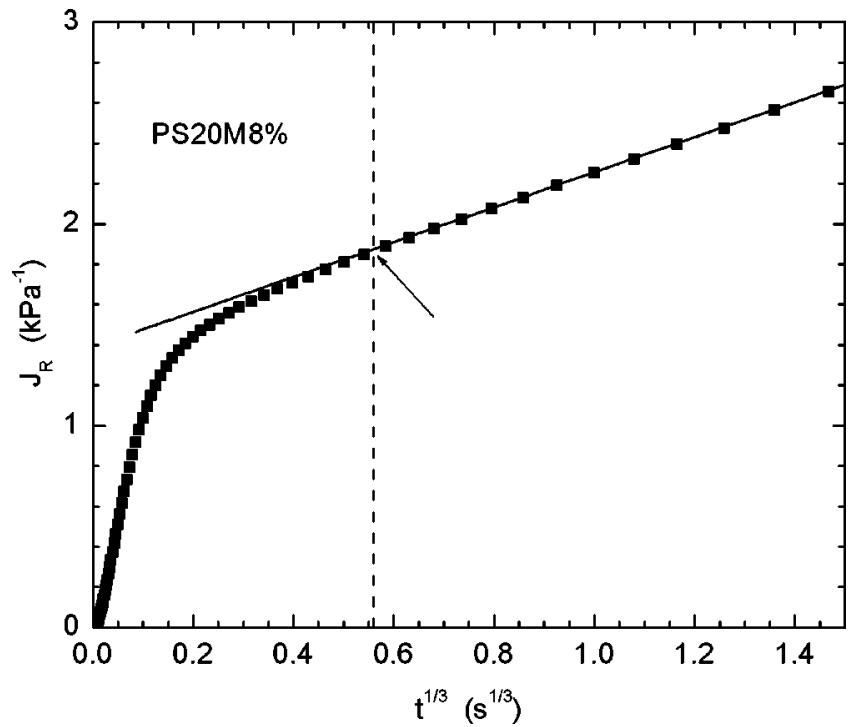

FIG. 4. Recoverable compliance calculated from the retardation spectrum in Fig. 3(b). The deviation at shorter times from the Andrade fit yields $\lambda_{e}$.

expected molecular weight independence. Although some of this discrepancy can be ascribed to the differences in magnitude between Rouse relaxation and retardation times, we believe the discrepancy is real, and reflects an outstanding issue in polymer viscoelasticity.

This work was supported by the Office of Naval Research and the National Science Foundation, Grant No. CTS0100579.

\section{References}

Andrade, E. N. da C., "On the viscous flow in metals, and allied phenomena," Proc. R. Soc. London, Ser. A 84, $1-12(1910)$.

Archer, L. A., "Separability criteria for entangled polymer liquids," J. Rheol. 43, 1555-1571 (1999).

Berry, G. C., "Thermal-mechanical studies on a heterocyclic polymer (BBB). Part 1: Tensile creep and recovery," J. Polym. Sci., Polym. Phys. Ed. 14, 451-478 (1976).

Berry, G. C., "Terminal retardation times and weights for the Rouse model for a crosslinked network," J. Polym. Sci., Part B: Polym. Phys. 25, 2203-2205 (1987).

Dean, G. D., B. E. Read, and P. E. Tomlins, "A model for long-term creep and the effects of physical aging in poly(butylene terephthalate)," Plast. Rubber Process. Applic. 13, 37-46 (1990).

Doi, M., and S. F. Edwards, The Theory of Polymer Dynamics (Oxford Science, New York, 1986).

Einaga, Y., K. Osaki, M. Kurata, S. Kimura, and M. Tamura, "Stress relaxation of polymer solutions under large strain," Polym. J. 2, 550-552 (1971).

Ferry, J. D. Viscoelastic Properties of Polymers (Wiley, New York, 1980).

Fetters, L. J., D. J. Lohse, S. T. Milner, and W. W. Graessley, "Packing length influence in linear polymer melts on the entanglement, critical, and reptation molecular weights," Macromolecules 32, 6847-6851 (1999).

Honerkamp, J., and J. Weese, "A nonlinear regularization method for the calculation of relaxation spectra," Rheol. Acta 32, 65-73 (1993).

Janeschitz-Kriegl, H. Polymer Melt Rheology (Springer-Verlag, New York, 1983).

Laun, H. M., "Relation between degree of orientation (double-refraction) and reversible deformation of drawn fused metals and calculation of strain using relaxation spectra," Colloid Polym. Sci. 259, 97-110 (1981).

Menezes, E. V., and W. W. Graessley, "Nonlinear rheological behavior of polymer systems for several shearflow histories," J. Polym. Sci., Polym. Phys. Ed. 20, 1817-1833 (1982). 
Mott, P. H., and C. M. Roland, "Birefringence of polymers in the softening zone," Macromolecules 31, 7095-7098 (1998).

Ngai, K. L., D. J. Plazek, and I. Echeverria, "Viscoelastic properties of amorphous polymers. 6: Local segmental contribution to the recoverable compliance of polymers," Macromolecules 29, 7937-7942 (1996).

Osaki, K., K. T. Inoue, and T. Isomura, "Stress overshoot of polymer solutions at high rates of shear," J. Polym. Sci., Part B: Polym. Phys. 38, 1917-1925 (2000).

Osaki, K., T. Inoue, T. Uematsu, and Y. Yamashita, "Evaluation methods of the longest rouse relaxation time of an entangled polymer in a semidilute solution," J. Polym. Sci., Part B: Polym. Phys. 39, 1704-1712 (2001).

Osaki, K., K. Nishizawa, and M. Kurata, "Material time constant characterizing the nonlinear viscoelasticity of entangled polymeric systems," Macromolecules 15, 1068-1071 (1982).

Okamoto, H., T. Inoue, and K. Osaki, "Birefringence of amorphous polymers 8. Viscoelasticity and birefringence of polyisoprene," J. Polym. Sci., Polym. Phys. Ed. 33, 417-424 (1995).

Plazek, D. J., "Dynamic mechanical and creep properties of a 23\% cellulose nitrate solution; Andrade creep in polymer systems," J. Colloid Sci. 15, 50-75 (1960).

Plazek, D. J., and V. M. O'Rourke, "Viscoelastic behavior of low molecular weight polystyrene," J. Polym. Sci., Polym. Phys. Ed. 9, 209-243 (1971).

Plazek, D. J., E. Riande, H. Markovitz, and N. Raghupathi, "Concentration dependence of the viscoelastic properties of polystyrene-tricresyl phosphate solutions,” J. Polym. Sci., Polym. Phys. Ed. 17, 2189-2213 (1979).

Plazek, D. J., "What's wrong with the moduli Charley Brown? or get the H out and go to L," J. Rheol. 36, $1671-1690$ (1992).

Plazek, D. J., C. A. Bero, G. Neumeister, G. Floudas, G. Fytas, and K. L. Ngai, "Viscoelastic properties of amorphous polymers 3: Low molecular weight poly(methyphenylsiloxane)," Colloid Polym. Sci. 272, 1430-1438 (1994).

Plazek, D. J., I.-C. Chay, K. L. Ngai, and C. M. Roland, "Thermorheological complexity of the softening dispersion in polyisobutylene," Macromolecules 28, $6432-6436$ (1995).

Plazek, D. J., “1995 Bingham Medal address: Oh, thermorheological simplicity, wherefore art thou?” J. Rheol. 40, 987-1014 (1996).

Plazek, D. J., and I. Echeverria, "Don't cry for me Charlie Brown, or with compliance comes comprehension," J. Rheol. 44, 831-841 (2000).

Read, B. E., "Viscoelastic behavior of amorphous polymers in the glass-rubber transition region-birefringence studies," Polym. Eng. Sci. 23, 835-843 (1983).

Roland, C. M., and P. H. Mott, "Response to comment on birefringence in the softening zone," Macromolecules 32, 4728-4728 (1999).

Roland, C. M., K. L. Ngai, P. G. Santangelo, X. H. Qiu, M. D. Ediger, and D. J. Plazek, "Temperature dependence of segmental and terminal relaxation in atactic polypropylene melts," Macromolecules 34, 6159-6160 (2001).

Sanchez-Reyes, J., and L. A. Archer, "Step shear dynamics of entangled polymer liquids,” Macromolecules 35, 5194-5202 (2002).

Sanchez-Reyes, J., and L. A. Archer, "Relaxation dynamics of entangled polymer liquids in steady shear flow," J. Rheol. 47, 469-482 (2003).

Santangelo, P. G., and C. M. Roland, "Temperature dependence of mechanical and dielectric relaxation in cis-1,4-polyisoprene," Macromolecules 31, 3715-3719 (1998).

Tao, H., C. Huang, and T. P. Lodge, "Correlation length and entanglement spacing in concentrated hydrogenated polybutadiene solutions," Macromolecules 32, 1212 (1999). 